\title{
Covid-19 e ageísmo: avaliação ética da distribuição de recursos em saúde
}

Tássia Salgado Soares ${ }^{1}$, Carla Corradi-Perini ${ }^{2}$, Caroline Perez Lessa de Macedo ${ }^{1}$, Uiara Raiana Vargas de Castro Oliveira Ribeiro ${ }^{2}$

1. Irmandade Hospital Santa Casa de Curitiba, Curitiba/PR, Brasil. 2. Pontifícia Universidade Católica do Paraná, Curitiba/PR, Brasil.

\begin{abstract}
Resumo
"Ageísmo" é o preconceito ou discriminação contra a pessoa idosa, seja por meio da estigmatização ou de práticas discriminatórias da sociedade e de suas instituições. No atual contexto da pandemia de covid-19, a postura ageísta da sociedade ocidental e, consequentemente, dos protocolos para distribuição de recursos em saúde tem sido fortemente evidenciada, trazendo consigo prejuízo importante à assistência a essa população. Este ensaio teórico discute manifestações e consequências do ageísmo em políticas de distribuição de recursos na pandemia, pensando as implicações bioéticas desse tipo de discriminação no que se refere aos princípios da justiça e da dignidade humana.
\end{abstract}

Palavras-chave: Infecções por coronavírus. Ageísmo. Idoso. Bioética. Políticas públicas de saúde.

\section{Resumen}

\section{Covid-19 y edadismo: evaluación ética de la distribución de los recursos sanitarios}

El "edadismo" se refiere al prejuicio y discriminación a las personas mayores, ya sea por estigmatización o prácticas discriminatorias por parte de la sociedad y sus instituciones. En el contexto actual de la pandemia de covid-19, se ha evidenciado fuertemente la postura edadista de la sociedad occidental y, en consecuencia, de los protocolos que involucran la distribución de los recursos en salud, trayendo consigo un daño importante a la atención en salud de esta población. Este ensayo teórico discute las manifestaciones y consecuencias del edadismo en el contexto de las políticas de distribución de recursos en salud en la pandemia, considerando las implicaciones éticas de esa discriminación respecto a los principios de justicia y dignidad humana.

Palabras clave: Infecciones por coronavirus. Edadismo. Anciano. Bioética. Políticas públicas de salud.

\begin{abstract}
Covid-19 and ageism: ethical assessment of health resources distribution

Ageism is the prejudice or discrimination of older adults, whether through stigmatization or discriminatory practices by society and its institutions. The current covid-19 pandemic context has shown Western society's ageist stance and, consequently, of its protocols on the distribution of health resources, leading to severe negative repercussions to the care of this population. This theoretical essay discusses the manifestations and consequences of ageism in the context of health resource distribution policies during the pandemic, considering the bioethical implications involved in this type of discrimination when considering the principles of justice and human dignity.
\end{abstract}

Keywords: Coronavirus infections. Ageism. Aged. Bioethics. Public health policy. 
"Ageísmo", termo derivado do inglês age (idade) + ism, foi cunhado em 1969 pelo médico psiquiatra e gerontólogo Robert Neil Butler ${ }^{1}$. Também conhecido como "etarismo" ou "idadismo", o termo refere-se ao preconceito e discriminação do indivíduo com base na idade, incluindo atitudes prejudiciais contra pessoas idosas e seu processo de envelhecimento, seja por meio de estigmatização e estereotipação ou práticas discriminatórias da sociedade e de suas instituições ${ }^{2,3}$.

Na sociedade ocidental, que valoriza o vigor da juventude, a aparência e o materialismo, o ageísmo encontra terreno fértil para se desenvolver ${ }^{1}$. Os valores culturais perpetuam o preconceito contra o idoso e a velhice, que é muitas vezes ridicularizada, desvalorizada e temida pelos jovens ${ }^{4}$. O estereótipo do idoso como pessoa frágil, dependente e improdutiva prevalece, desconsiderando a heterogeneidade do envelhecimento.

$\mathrm{Na}$ atual crise da covid-19, há uma ênfase indevida no conceito de idade cronológica, em detrimento da noção de idade biológica, relacionada à funcionalidade e ao grau de conservação do indivíduo, sem relação direta com a idade em anos $^{5}$. Essa ênfase generaliza a população geriátrica e culmina com a desvalorização da vida da pessoa idosa ${ }^{6-8}$. Assim, a pandemia escancara o ageísmo, que sempre esteve presente na realidade social, mas, como outras práticas discriminatórias existentes, manifestava-se na maioria das vezes de forma velada ${ }^{9,10}$.

Portanto, é importante discutir o conceito de ageísmo e suas implicações bioéticas, identificar atitudes ageístas - tanto em nível individual como em sociedade - e suas consequências e combater políticas públicas discriminatórias, lutando pela solidariedade entre gerações. Neste artigo, discute-se o ageísmo especificamente no âmbito da distribuição de recursos em saúde. O objetivo é pensar as implicações bioéticas desse tipo de discriminação no que se refere aos princípios da justiça e da dignidade humana, considerando o cenário global de saúde e a atual de pandemia de covid-19. Para fundamentar a discussão, realizou-se pesquisa documental sobre os temas covid-19, ageísmo e alocação de recursos em saúde. A análise crítica da literatura encontrada, nacional e internacional, fundamentou este ensaio teórico.

\section{Ageísmo na assistência à saúde}

Para avaliar a repercussão do ageísmo no contexto da pandemia de covid-19, é necessário compreender a história desse tipo de discriminação na assistência à saúde. Dentre os profissionais da área, assim como nos demais setores da sociedade, atitudes ageístas são frequentes e impactam o cuidado à pessoa idosa ${ }^{4}$. É comum, por exemplo, que cirurgias sejam contraindicadas a idosos sem considerar as chances reais de sucesso do procedimento ${ }^{11}$. A idade cronológica também parece influenciar profissionais médicos em decisões sobre ordem de não reanimação cardiopulmonar ${ }^{12}$.

O ageísmo na assistência à saúde inclui ainda menor oferta de diagnósticos e tratamentos, motivada muitas vezes pela concepção de que as queixas do idoso decorrem do processo natural de senescência e não merecem ser investigadas ${ }^{9,11}$. Na pesquisa, em ensaios clínicos, a faixa etária dos mais velhos é raramente incluída, seja por falta de interesse ou pela heterogeneidade dessa população ${ }^{11}$. Portanto, o cuidado à pessoa idosa sofre clara e prejudicial interferência do ageísmo.

\section{Ageísmo na pandemia de covid-19}

O surto inicial de Sars-CoV-2 ocorreu na China, onde se observou que $20 \%$ das mortes ocorriam em pessoas acima de 60 anos e que a letalidade aumentava com a idade, chegando a $18 \%$ em maiores de 80 anos $^{13,14}$. Ficou evidente, portanto, que a população geriátrica era um grupo de risco para a covid-19. Com a epidemia alçada ao status de pandemia, autoridades globais de saúde e governos emitiram alertas à população idosa quanto ao risco aumentado de doença grave e fatal associada ao novo coronavírus, reforçando a necessidade de isolamento social para esse grupo, o que acabou por fomentar discursos e atitudes ageístas ${ }^{15}$.

A discussão sobre ageísmo no contexto de covid-19 veio à tona mais fortemente com o colapso do sistema de saúde da Itália, país com a população mais idosa da Europa ${ }^{16}$. Devido ao cenário crítico, com altos níveis de infecção e escassez de recursos, foi necessário que profissionais de saúde priorizassem pacientes em detrimento de outros ${ }^{17}$. 0 primeiro protocolo de alocação de recursos escassos na pandemia de covid-19 foi emitido pela Società 
Italiana di Anestesia, Analgesia, Rianimazione e Terapia Intensiva (Siaarti). Uma das medidas propostas para limitar a admissão em terapia intensiva foi a idade cronológica - em conjunto com a presença de comorbidade e status funcional -, o que provocou discussões bioéticas sobre parâmetros de triagem para alocação de recursos ${ }^{18}$.

A população idosa é erroneamente representada como um grupo homogêneo e vulnerável de pessoas dependentes, frágeis e incapazes de contribuir com a sociedade. Essa visão, propagada pela imprensa, instituições governamentais, políticos e mídias sociais, desconsidera a heterogeneidade do envelhecimento, as contribuições sociais da pessoa idosa e seu valor como indivíduo ${ }^{6,7,9,19}$. O ageísmo cria, portanto, uma divisão entre jovens e pessoas idosas, enfatizando - ainda mais no caso da covid-19 - a suscetibilidade dos mais velhos. Dessa forma, pessoas jovens tendem a pensar que estão imunes ao vírus, o que pode levar a comportamentos de risco e tensões intergeracionais, como discursos de ódio direcionados aos idosos ${ }^{6,10}$.

Tanto nos meios de comunicação como nas políticas e recomendações em saúde, foca-se a pessoa idosa como grupo mais impactado pela pandemia, desconsiderando comorbidades como doença cardíaca, asma, diabetes, tabagismo e obesidade ${ }^{8,9,19}$. Definir grupos com base apenas na idade cronológica, subestimando outras diferenças internas, é uma suposição ageísta, que fomenta preconceito, estereótipos e discriminação ${ }^{6,10}$. Há relatos de pessoas com mais de 100 anos que se recuperaram da covid-19, assim como têm ocorrido óbitos pela doença entre jovens adultos, o que reforça a insuficiência da idade cronológica como critério único para estabelecer prioridades de assistência em saúde ${ }^{7}$.

Com a disseminação do Sars-CoV-2 por todo o mundo, foram criados diversos protocolos de alocação de recursos em saúde escassos. O documento já mencionado da Siaarti usava o conceito de "anos de vida salvos" e considerava os gastos empregados em pessoas previamente hígidas para justificar a discriminação etária ${ }^{18}$. A idade cronológica continuou sendo utilizada como critério em políticas promulgadas em outros países da Europa, como a Suíça, e nos Estados Unidos, especialmente em protocolos de priorização de recursos e triagem para terapia intensiva, muitas vezes de forma arbitrária, sem que houvesse padronização de pontos de corte de idade ${ }^{10,20-22}$.
No Brasil, a Associação de Medicina Intensiva Brasileira (Amib) e a Associação Brasileira de Medicina de Emergência (Abramede) foram as primeiras a publicar um protocolo de alocação de recursos, elencando como um dos três critérios principais de triagem a idade cronológica ${ }^{23}$. A proposta ia de encontro ao artigo 9 da Resolução 2.156/2016 do Conselho Federal de Medicina (CFM), que estabelece os critérios de admissão e alta em unidade de terapia intensiva: As decisões sobre admissão e alta em unidade de tratamento intensivo (UTI) devem ser feitas de forma explícita, sem discriminação por questões de religião, etnia, sexo, nacionalidade, cor, orientação sexual, idade, condição social, opinião política, deficiência, ou quaisquer outras formas de discriminação ${ }^{24}$.

Após críticas, a Amib e a Abramede publicaram uma segunda versão do protocolo, juntamente com a Sociedade Brasileira de Geriatria e Gerontologia (SBGG) e a Academia Nacional de Cuidados Paliativos (ANCP). No novo documento, as entidades assumem que o critério etário poderia ser discriminatório e inconstitucional e sugerem incluir na equipe de triagem, sempre que possível, um bioeticista e um representante da comunidade ${ }^{25}$.

A American Geriatrics Society (AGS), em seu posicionamento oficial sobre alocação de recursos, mostrou preocupação com estratégias adotadas em estados americanos que consideraram a idade como critério de triagem. Para a AGS, esse critério violava o princípio bioético da justiça, uma vez que tem vieses implícitos e, portanto, é discriminatório. A instituição recomenda que pontos de corte etários nunca sejam usados como critério categórico de exclusão de intervenções terapêuticas ${ }^{26}$.

A pandemia de covid-19 expôs o ageísmo latente na sociedade, potencializando discursos, atitudes e ações discriminatórias em todos os níveis - desde a população em geral até líderes mundiais e instituições. O ageísmo manifesta-se também em políticas de distribuição de recursos e assistência em saúde no contexto da pandemia por covid-19 que estabelecem a idade cronológica como critério único para determinar vulnerabilidades, prognósticos e opções de tratamento. Essa perspectiva desconsidera a heterogeneidade da população idosa, seus valores e preferências. Portanto, o presente artigo questiona a eticidade do critério etário e destaca a necessidade de otimizar recursos no cenário crítico da pandemia ${ }^{6,7,10,17,18}$. 
Aspecto antiético do ageísmo na alocação de recursos em saúde

De acordo com a bioética principialista ${ }^{27}$, utilizada como referência na construção do Código de Ética Médica (CEM) brasileiro ${ }^{28}$, o princípio da justiça propõe que se dê ao indivíduo o que lhe é devido - seus direitos, portanto. Nesse sentido, a justiça distributiva busca a alocação justa e equitativa dos recursos, dentre eles a saúde, conforme as normas que estruturam a dinâmica do meio social. No entanto, não há um conceito único de justiça, capaz de abarcar todos os conflitos relacionados à distribuição dos recursos, o que leva a diferentes interpretações sobre como aplicar esse princípio ${ }^{27,29}$.

Há diversas teorias que visam aplicar a justiça distributiva na saúde. O liberalismo prioriza a liberdade econômica e social na distribuição de recursos. Já o igualitarismo defende o acesso equânime aos bens, conforme a necessidade do indivíduo. O utilitarismo, por sua vez, propõe reunir variados critérios de justiça a fim de otimizar a distribuição ${ }^{27}$. Cada uma a seu modo, tais teorias buscam definir o conceito de justiça considerando a finitude dos recursos em saúde. Criam-se, assim, embates sobre proporcionalidade que incluem não apenas a questão da prevenção e promoção da saúde versus assistência à saúde, como também a definição de quais grupos sociais deveriam ser ou não beneficiados pela destinação dos recursos ${ }^{27,29}$.

A discriminação da pessoa idosa, ou ageísmo, na maioria das vezes não é contemplada pelas teorias de justiça distributiva. No entanto, dado o crescimento exponencial da população idosa em todo o mundo, a segregação desse grupo no acesso à assistência tem se acentuado. Tal segregação se baseia na ideia preconceituosa de que idosos utilizam mais recursos em decorrência de doenças, mas não contribuem tanto para o sistema de saúde, do ponto de vista socioeconômico, se comparados aos mais jovens.

Do ponto de vista da teoria da equidade, o direito à saúde, com igualdade de acesso, deveria minimizar o desfavorecimento da pessoa em relação ao acaso natural ou às circunstâncias sociais, priorizando um mínimo a ser ofertado e a equanimidade de oportunidades ${ }^{27,29}$. No entanto, alguns argumentos baseados nessa teoria recorrem à "oportunidade equitativa" para justificar o critério. Tais argumentos defendem uma "duração normal da vida" (todos deveriam ter oportunidade similar de passar por diferentes fases), enfatizam o custo aumentado de prolongar a vida da pessoa idosa (em detrimento da pessoa jovem, cujo tratamento seria menos custoso e teria maior potencial em relação a tempo de vida) e apontam o baixo sucesso terapêutico de medidas instituídas nessa faixa etária (em comparação com indivíduos mais jovens) ${ }^{27,30}$.

O caráter antiético da discriminação relacionada à idade cronológica tem ainda uma face institucional. Nesse caso, o ageísmo é diluído em regras ou procedimentos sociais ${ }^{30}$. Essa institucionalização se revela quando consideramos que os valores éticos de uma sociedade atuam diretamente como base da formulação de políticas de saúde e distribuição de recursos ${ }^{29}$.

Mesmo um órgão como a Organização Mundial da Saúde (OMS) tem perpetuado o ageísmo em seus planos de ação, influenciando diretamente a pesquisa e as políticas de saúde em todo o mundo. O conceito de "ano de vida ajustado por incapacidade" foi definido em 1993 para estudar o impacto de doenças e fundamentar o planejamento de políticas relacionadas à saúde. Em seu cálculo, o conceito incorporava a medida de "anos perdidos de vida", ajustada de acordo com uma idade arbitrária de máximo de anos a serem vividos - algo entre 65 e 80 anos. Dessa forma, a incapacidade era valorizada de forma desproporcional entre jovens e idosos, pois esses últimos eram previamente julgados por uma maior dependência social supostamente inerente à faixa etária. A medida foi interrompida apenas em $2010^{30}$.

No período entre 2008 e 2013, a OMS trabaIhou com o conceito de "morte prematura" no plano global de abordagem de doenças crônicas não transmissíveis (como hipertensão arterial sistêmica e diabetes mellitus). De modo arbitrário, o plano estabelecia como precoce a morte antes dos 70 anos, justificando que valorizar a assistência à população mais jovem futuramente também beneficiaria as pessoas idosas ${ }^{30}$.

Outro exemplo de ageísmo é a medida de anos de vida ajustados por qualidade (Avaq), utilizada nas análises de custo-utilidade por instituições regulamentadoras de recursos em saúde, a exemplo do National Institute for Health and Clinical Excellence (Nice), na Inglaterra. O cálculo 
se baseia em anos de expectativa de vida associados a um valor numérico arbitrário para qualidade de vida, classificando como de baixa prioridade os tratamentos que tenham menor impacto na expectativa de vida ${ }^{31}$.

Tais políticas discriminam a população idosa e negligenciam uma série de desafios: multimorbidade, cuidados paliativos ou mesmo doenças transmissíveis como o vírus da imunodeficiência humana. Indiretamente, valoriza-se mais a saúde do indivíduo em faixa etária economicamente produtiva e com uma hipotética maior expectativa de vida. Ao determinar quantos e quais são os "anos de vida válidos" a serem prolongados, os argumentos limitam o valor da vida à produção de riquezas, rotulando os idosos como "economicamente improdutivos". No entanto, é o próprio ageísmo que na verdade contribui para o isolamento da pessoa idosa do mercado de trabalho e do convívio social, aprofundando sistematicamente a "improdutividade". Assim, mesmo teorias que consideram o conceito de equidade de oportunidade acabam corroborando políticas ageístas na saúde ${ }^{32}$.

$\mathrm{O}$ ageísmo institucional presente nas políticas de saúde é também preocupante quando se considera que cerca de dois terços da população mundial de idosos está alocada em países subdesenvolvidos e em desenvolvimento ${ }^{30}$, onde recursos financeiros são geralmente escassos e, portanto, requerem uma lógica utilitarista de distribuição. No Brasil, o direito à saúde é garantido pela Constituição Federal de $1988^{33}$ e a pela Lei 8.080/1990 ${ }^{34}$, conhecida como Lei Orgânica da Saúde, que regulamenta o Sistema Único de Saúde (SUS). A saúde é considerada direito de todo cidadão brasileiro, e o SUS tem por princípios a universalidade de acesso e a integralidade e igualdade da assistência, não cabendo preconceitos de qualquer espécie ${ }^{34}$. Assim, em nosso país, o Estado incorpora o igualitarismo e assume a responsabilidade de promover o acesso à saúde e defender a dignidade humana, partindo da premissa de que se deve garantir a justiça distributiva conforme as necessidades de cada indivíduo, com o fim de minimizar desigualdades, sejam biológicas ou sociais ${ }^{29}$.

A política de saúde brasileira difere da praticada em outros países, como os Estados Unidos, onde o acesso à saúde não se dispõe a ser igualitário e pretere segmentos socioeconomicamente vulneráveis da população sob a justificativa do liberalismo e das regras do livre mercado ${ }^{29}$. O Estado brasileiro abraça a dignidade humana como um de seus princípios fundamentais, conforme descrito no artigo $1^{\circ}$ da Constituição de 1988, determinando ainda a defesa da dignidade, do bem-estar e do direito à vida da pessoa idosa em seu artigo $230^{33}$. Dessa forma, fica explícito na legislação o direcionamento em prol da dignidade da pessoa idosa, incluindo o acesso à saúde, que é, portanto, um direito estabelecido.

\section{Aspecto antiético do ageísmo na pandemia de covid-19}

No atual contexto de pandemia, protocolos de instituições brasileiras como Amib, Abramede (em sua primeira versão) e Hospital Albert Einstein propuseram a idade cronológica, de forma explícita ou velada (como "anos de vida a serem salvos"), como critério isolado para destinar recursos escassos, principalmente em situações que envolvem o acesso do paciente a UTI e a medidas de suporte avançado de vida (SAV) ${ }^{35,36}$. Tal postura não difere de protocolos propostos em países desenvolvidos que sentiram fortemente o impacto do conflito entre a alta demanda e a escassez de recursos, como Itália ${ }^{17}$, Espanha ${ }^{37}$ e Suíça ${ }^{22}$. No entanto, propor que o direito de um jovem receber ventilação mecânica prevaleça por conta de uma possível maior expectativa de vida é minimizar o direito à vida e à dignidade humana da pessoa idosa, desconsiderando o benefício de medidas intervencionistas em idosos com boa reserva funcional e, portanto, boas perspectivas de recuperação.

Dessa forma, argumentos que utilizam como justificativa a baixa probabilidade de recuperação do idoso infectado pelo coronavírus não levam em consideração uma das principais características do envelhecimento: a heterogeneidade no que se refere a funcionalidade e status de saúde ${ }^{38}$. Pessoas idosas, ainda que da mesma faixa etária, são distintas entre si, e podem ou não apresentar condições que impliquem maior morbimortalidade por covid-19, como ocorre também, por exemplo, na síndrome de fragilidade ${ }^{17,38,39}$. Ou seja, é perfeitamente possível que um idoso com bom status funcional e boas condições de saúde se beneficie de medidas de SAV; não há relação direta com a idade cronológica. 
Indo além da análise biológica, Dias e Gonçalves ${ }^{40}$ questionam até onde é correto considerar o "tempo de vida" sob a lógica equitativa, visto que isso sobreporia o direito da pessoa jovem ao da pessoa idosa a partir de uma métrica arbitrária, que desconsidera o conteúdo - isto é, a biografia - e o valor que a pessoa atribui a sua vida. Nesse sentido, em maio de 2020, a Sociedade Brasileira de Bioética (SBB) chamou atenção para aspectos éticos do enfrentamento da pandemia no que concerne à alocação de recursos e ao uso igualitário de tecnologias em saúde. Já em seu início, a Resolução SBB 1/2020 considera que o respeito à dignidade humana deve ser o fundamento maior para as tomadas de decisão e condutas em saúde sem qualquer distinção que importe na desvalorização e discriminação de pessoas, comunidades ou grupos socialmente vulneráveis ${ }^{41}$.

Do ponto de vista bioético, o ageísmo manifesto no critério da idade cronológica para instituição de SAV e internação em UTI transgride tanto o princípio da dignidade humana quanto da beneficência, pois limita o acesso a medidas potencialmente benéficas à pessoa idosa infectada pelo coronavírus. Por isso a SBB mostra preocupação quanto a protocolos de acesso à UTI que estabelecem a faixa etária como critério. Em direção oposta a tais protocolos, a instituição reforça o respeito à dignidade humana, garantido constitucionalmente, como norteador da tomada de decisão, a fim de que não se incorra em discriminação na assistência em saúde.

No entanto, persiste a necessidade - ainda mais crítica em contextos como o atual - de distribuir os recursos de modo a beneficiar o maior número de indivíduos. Mas como fazê-lo de forma ética, sem excluir a pessoa idosa? Essa tarefa é impossível caso o protocolo não abarque o princípio da dignidade humana. Reconhecer o indivíduo como único, portador de valor essencial e intrínseco, fim em si mesmo e não meio, deve ser a orientação fundamental de qualquer recomendação ${ }^{42}$. Esse reconhecimento envolve afirmar o direito à saúde da pessoa idosa, reconhecendo seus valores, sua biografia e sua relevância para a sociedade.

Em geral, protocolos que adotam algoritmos respaldados cientificamente para detectar condições clínicas indicativas de pior prognóstico ou menor benefício em medidas de SAV, independentemente da faixa etária do paciente, permitem decisões mais assertivas ${ }^{17,37,41,42}$. Condições como fragilidade, multimorbidade e status funcional devem ser rastreadas e incorporadas na tomada de decisão, prevalecendo sobre a idade cronológica isolada ${ }^{17,42}$, visto que por meio delas é possível identificar pacientes para os quais o SAV não será benéfico (podendo até ser prejudicial, tendo em vista o risco de distanásia). O respeito às diretivas antecipadas de vontade, caso o paciente as tenha registrado, também é fundamental nesse processo. Desse modo é possível alocar recursos escassos de forma mais digna, justa e beneficente, respeitando a autonomia do indivíduo.

\section{Considerações finais}

Até então presente de forma velada no meio social e em certas políticas de saúde, o ageísmo revela-se por completo na pandemia de covid-19, seja na postura discriminatória da sociedade ao rotular idosos como grupo de risco, seja nos protocolos de destinação de recursos que utilizam a idade cronológica como medida arbitrária para a tomada de decisão. No entanto, em tempos normais ou de pandemia, não há justificativa para o ageísmo. Além de moralmente defensáveis, o direito à saúde e à dignidade humana é garantido constitucionalmente no Brasil, e do ponto de vista bioético qualquer tipo de discriminação na distribuição dos recursos sanitários é repreensível.

Além de suprimirem a oportunidade de o idoso se beneficiar de medidas terapêuticas (incluindo SAV), restrições baseadas apenas na idade cronológica reforçam a discriminação a esse grupo e reduzem a vida de um indivíduo a números arbitrários, que desconsideram valores e escoIhas. Assim, apesar de já haver diversas recomendações éticas visando protocolos mais justos de alocação de recursos, ainda é fundamental educar profissionais de saúde - não somente geriatras/ gerontólogos - para reconhecer o ageísmo institucional. Estender e aprofundar a discussão e o conhecimento sobre a pessoa idosa e suas particularidades é essencial para combater esse tipo de discriminação. 


\section{Referências}

1. Butler RN. Age-ism: another form of bigotry. Gerontologist [Internet]. 1969 [acesso 27 ago 2020];9(4):243-6. DOI: 10.1093/geront/9.4_Part_1.243

2. Ayalon L, Tesch-Römer C. Contemporary perspectives on ageism. Cham: Springer International Publishing; 2018.

3. Officer A, de la Fuente-Núñez V. A global campaign to combat ageism. Bull World Health Organ [Internet]. 2018 [acesso 27 ago 2020];96(4):295-6. DOI: 10.2471/BLT.17.202424

4. Nelson TD. Reducing ageism: which interventions work? Am J Public Health [Internet]. 2019 [acesso 27 ago 2020];109(8):1066-7. DOI: 10.2105/AJPH.2019.305195

5. Freitas EV, Py L. Tratado de geriatria e gerontologia. $4^{\mathrm{a}}$ ed. Rio de Janeiro: Guanabara Koogan; 2016.

6. Ayalon L, Chasteen A, DiehI M, Levy BR, Neupert SD, Rothermund $K$ et al. Aging in times of the covid-19 pandemic: avoiding ageism and fostering intergenerational solidarity. J Gerontol B Psychol Sci Soc Sci [Internet]. 2020 [acesso 27 ago 2020];20(20):1-4. DOI: 10.1093/geronb/gbaa051

7. Ayalon L. There is nothing new under the sun: ageism and intergenerational tension in the age of the covid-19 outbreak. Int Psychogeriatrics [Internet]. 2020 [acesso 27 ago 2020];32(20):1221-4. DOI: 10.1017/ S1041610220000575

8. Ehni HJ, Wahl HW. Six propositions against ageism in the covid-19 pandemic. J Aging Soc Policy [Internet]. 2020 [acesso 27 ago 2020];32(4-5):515-25. DOI: 10.1080/08959420.2020.1770032

9. Reynolds L. The covid-19 pandemic exposes limited understanding of ageism. J Aging Soc Policy [Internet]. 2020 [acesso 27 ago 2020];32(4-5):499-505. DOI: 10.1080/08959420.2020.1772003

10. Previtali F, Allen LD, Varlamova M. Not only virus spread: the diffusion of ageism during the outbreak of covid-19. J Aging Soc Policy [Internet]. 2020 [acesso 27 ago 2020];32(4-5):506-14. DOI: 10.1080/08959420.2020.1772002

11. Holmes E, Crome P, Arora A. Patients' preferences and existential perspective: what to consider and how should patient's expectations be guided? Aging Clin Exp Res [Internet]. 2018 [acesso 27 ago 2020];30(3):271-5. DOI: 10.1007/s40520-017-0868-7

12. Cook I, Kirkup AL, Langham LJ, Malik MA, Marlow G, Sammy I. End of life care and do not resuscitate orders: how much does age influence decision making? A systematic review and meta-analysis. Gerontol Geriatr Med [Internet]. 2017 [acesso 27 ago 2020];3. DOI: 10.1177/2333721417713422

13. Wu Z, McGoogan JM. Characteristics of and important lessons from the coronavirus disease 2019 (covid-19) outbreak in China. JAMA [Internet]. 2020 [acesso 27 ago 2020];323(13):1239-42. DOI: 10.1001/jama.2020.2648

14. Lai CC, Shih TP, Ko WC, Tang HJ, Hsueh PR. Severe acute respiratory syndrome coronavirus 2 (SARS-CoV-2) and coronavirus disease-2019 (covid-19): the epidemic and the challenges. Int J Antimicrob Agents [Internet]. 2020 [acesso 27 ago 2020];55(3). DOI: 10.1016/j.ijantimicag.2020.105924

15. Brooke J, Jackson D. Older people and covid-19: isolation, risk and ageism. J Clin Nurs [Internet]. 2020 [acesso 27 ago 2020];29(13-14):2044-6. DOI: 10.1111/jocn.15274

16. Petretto DR, Pili R. Ageing and covid-19: what is the role for elderly people? Geriatrics [Internet]. 2020 [acesso 27 ago 2020];5(2):25. DOI: 10.3390/geriatrics5020025

17. Cesari M, Proietti M. covid-19 in Italy: ageism and decision making in a pandemic. J Am Med Dir Assoc [Internet]. 2020 [acesso 27 ago 2020];21(5):576-7. DOI: 10.1016/j.jamda.2020.03.025

18. Riccioni L, Bertolini G, Giannini A, Gristina GR, Livigni S, Mistralettiet G et al. Clinical ethics recommendations for the allocation of intensive care treatments, in exceptional, resource-limited circumstances. Recenti Prog Med [Internet]. 2020 [acesso 27 ago 2020];111(4):207-11. DOI: 10.1701/3347.33183

19. Fraser S, Lagacé M, Bongué B, Ndeye N, Guyot J, Bechard L et al. Ageism and covid-19: what does our society's response say about us? Age Ageing [Internet]. 2020 [acesso 27 ago 2020];49(5):692-5. DOI: 10.1093/ageing/afaa097 
20. Timothy WF, Ferrante LE, Brown T, Francis L, Widera E, Rhodes $\mathrm{R}$ et al. AGS position statement: resource allocation strategies and age-related considerations in the covid-19 era and beyond. J Am Geriatr Soc [Internet]. 2020 [acesso 27 ago 2020];68(6):1136-42. DOI: 10.1111/jgs.16537

21. Antommaria AHM, Gibb TS, McGuire AL, Wolpe RP, Wynia MK, Applewhite MK et al. Ventilator triage policies during the covid-19 pandemic at U.S. hospitals associated with members of the Association of Bioethics Program Directors. Ann Intern Med [Internet]. 2019 [acesso 27 ago 2020];173:188-94. DOI: 10.7326/M20-1738

22. Swiss Academy of Medical Sciences. covid-19 pandemic: triage for intensive-care treatment under resource scarcity. Swiss Med Wkly [Internet]. 2020 [acesso 27 ago 2020];150. DOI: 10.4414/smw.2020.20229

23. Kretzer L, Berbigier E, Lisboa R, Grumann AC, Andrade J. Protocolo AMIB de alocação de recursos em esgotamento durante a pandemia por covid-19 [Internet]. São Paulo: Associação de Medicina Intensiva Brasileira; 2020 [acesso 27 ago 2020]. Disponível: https://bit.ly/3sJyxUv

24. Conselho Federal de Medicina. Resolução CFM n 2.156/2016. Estabelece os critérios de admissão e alta em unidade de terapia intensiva [Internet]. Brasília: CFM; 2016 [acesso 27 ago 2020]. Disponível: https://bit.ly/3gAqh71

25. Associação de Medicina Intensiva Brasileira. Recomendações da AMIB, ABRAMEDE, SBGG e ANCP de alocação de recursos em esgotamento durante a pandemia por covid-19 [Internet]. São Paulo: Associação de Medicina Intensiva Brasileira; 2020 [acesso 27 ago 2020]. Disponível: https://bit.ly/3vgoDvu

26. Farrell TW, Francis L, Brown T, Ferrante LE, Widera E, Rhodes $R$ et al. Rationing limited healthcare resources in the covid-19 era and beyond: ethical considerations regarding older adults. J Am Geriatr Soc [Internet]. 2020 [acesso 27 ago 2020];68(6):1143-9. DOI: 10.1111/jgs.16539

27. Beauchamp TL, Childress JF. Princípios de ética biomédica. $2^{\text {a }}$ ed. São Paulo: Loyola; 2011.

28. Soares FJP, Shimizu HE, Garrafa V. Código de Ética Médica brasileiro: limites deontológicos e bioéticos. Rev. bioét. (Impr.). [Internet]. 2017 [acesso 27 ago 2020];25(2):244-54. DOI: 10.1590/1983-80422017252184

29. Antonio $P$, Fortes DC. Orientações bioéticas de justiça distributiva aplicada às ações e aos sistemas de saúde. Rev. bioét. (Impr.). [Internet]. 2008 [acesso 27 ago 2020];16(1):25-39. Disponível: https://bit.ly/3epHOXW

30. Lloyd-Sherlock PG, Ebrahim S, McKee M, Prince MJ. Institutional ageism in global health policy. BMJ [Internet]. 2016 [acesso 27 ago 2020];354. DOI: 10.1136/bmj.i4514

31. Harris J, Regmi S. Ageism and equality: Great Britain [Internet]. London: Age UK; 2018 [acesso 27 ago 2020]. Disponível: https://bit.ly/3xmLSWk

32. Tarazona-Santabalbina FJ, Martínez-Velilla N, Vidán MT, García-Navarro JA. covid-19, adulto mayor y edadismo: errores que nunca han de volver a ocurrir. Rev Esp Geriatr Gerontol [Internet]. 2020 [acesso 27 ago 2020];55(4):191-2. Disponível: https://bit.ly/3vjJn5B

33. Brasil. Presidência da República. Constituição da República Federativa do Brasil. Diário Oficial da União [Internet]. Brasília, 5 out 1988 [acesso 27 ago 2020]. Disponível: https://bit.ly/2QScy0u

34. Brasil. Presidência da República. Lei ${ }^{\circ} 8.080$, de 19 de setembro de 1990 . Dispõe sobre as condições para a promoção, proteção e recuperação da saúde, a organização e o funcionamento dos serviços correspondentes e dá outras providências. Diário Oficial da União [Internet]. Brasília, 19 set 1990 [acesso 27 ago 2020]. Disponível: https://bit.ly/3gAqgQk

35. Costa A, Maria C, Costa A. Protocolos em tempos de pandemia por covid-19 com critérios para alocação de recursos escassos: podem considerá-los (in)justos? Diversitates [Internet]. 2020 [acesso 27 ago 2020];12(2):131-62. Disponível: https://bit.ly/2QP8LkA

36. Satomi E, Souza PMR, Thomé BC, Reingenheim C, Werebe E, Troster EJ et al. Fair allocation of scarce medical resources during covid-19 pandemic: ethical considerations. Einstein (São Paulo) [Internet]. 2020 [acesso 27 ago 2020];18. DOI: 10.31744/einstein_journal/2020ae5775

37. Herreros B, Gella P, Real de Asua D. Triage during the covid-19 epidemic in Spain: better and worse ethical arguments. J Med Ethics [Internet]. 2020 [acesso 27 ago 2020];46:455-8. DOI: 10.1136/ medethics-2020-106352 
38. Rosen T, Ferrante LE, Liu SW, Benton EA, Mulcare MR, Stern ME et al. Managing older adults with presumed covid-19 in the emergency department: a rational approach to rationing. J Am Geriatr Soc [Internet]. 2020 [acesso 27 ago 2020];68:1631-5. DOI: 10.1111/jgs.16651

39. Perez MP. Is frailty and not chronological age the essential prognostic element in older patients with covid-19? Geriatr Gerontol Aging [Internet]. 2020 [acesso 27 ago 2020];14(2):77-8. DOI: 10.5327/Z24472123202020v14n2EDT1

40. Dias MC, Gonçalves L. Escolha sobre quem deve viver: bioética e covid-19 no contexto brasileiro. Brasil de Fato [Internet]. Opinião; 28 abr 2020 [acesso 27 ago 2020]. Disponível: https://bit.ly/3aD4Qyu

41. Sociedade Brasileira de Bioética. Recomendação SBB $n^{\circ}$ 01/2020. Define os aspectos éticos no enfrentamento da covid-19, em defesa dos mais vulneráveis e do acesso igualitário aos leitos de UTI [Internet]. Brasília: Sociedade Brasileira de Bioética; 2020 [acesso 27 ago 2020]. Disponível: https://bit.ly/3vipqvE

42. Maglio I, Valdez P, Cámera L, Finn B, Klein M, Pincemin I et al. Guías éticas para la atención durante la pandemia covid-19: recomendaciones multisocietarias para asignación de recursos. Medicina (B Aires) [Internet]. 2020 [acesso 27 ago 2020];80:45-64. Disponível: https://bit.ly/3dL6H6j

Tássia Salgado Soares - Especialista - tassiasoares@hotmail.com

(D) 0000-0001-8684-0913

Carla Corradi-Perini - Doutora - carla.corradi@pucpr.br

(D) 0000-0002-9340-8704

Caroline Perez Lessa de Macedo - Especialista - carol_plessa@hotmail.com

(D) 0000-0002-9511-7759

Uiara Raiana Vargas de Castro Oliveira Ribeiro - Mestre - uiaravargasribeiro@gmail.com

(D) 0000-0002-1624-2245

Correspondência

Uiara Raiana Vargas de Castro Oliveira Ribeiro - Pontifícia Universidade Católica do Paraná.

Escola de Medicina. Rua Imaculada Conceição, 1115 CEP 80215-901. Curitiba/PR, Brasil.

Participação das autoras

Tássia Salgado Soares e Uiara Raiana Vargas de Castro Oliveira Ribeiro realizaram a revisão bibliográfica, redigiram o artigo e, junto com Caroline Perez Lessa de Macedo, conceberam o estudo. Carla Corradi-Perini contribuiu com a revisão crítica da versão final do artigo.

Recebido: 25.8 .2020

Revisado: 4.2 .2021

Aprovado: 10.2.2021 